\title{
GROWTH HORMONE MODULATES THE INFLAMMATORY AND APOPTOTIC PATHWAYS INCORPORATED IN FLUOROURACIL- INDUCED ORAL MUCOSITIS IN RATS
}

\author{
Rasha H. Al Serwi*, Samar F. Darwish ${ }^{* *}$ and Yasmen F. Mahran***
}

\begin{abstract}
Introduction: Oral mucositis (OM) is a well-known complication of radiotherapy (RT) and chemotherapy (CT) in cancer patients. Although, 5-flourouracil (5-FU) is one of the standard cytotoxic therapies, it is one of the most common causes of OM, which results in delay, dose reduction or treatment discontinuation, thus this intensifies the need for an effective chemoprotective agent. We aimed to investigate the potential chemoprotective effect of growth hormone (GH) on 5-FU-induced $\mathrm{OM}$ in rats.
\end{abstract}

Material \& Methods: Rats were either exposed to a single 5-FU injection $(160 \mathrm{mg} / \mathrm{kg}$ ip) and/or treated with GH (1 mg/kg s.c). Oxidative stress, inflammatory and apoptotic markers were assessed.

Results: An array of mucosal damage was produced as a result of 5-FU injection, which was evident by histopathology. 5-FU induced a remarkable increase in lipid peroxidation accompanied with a significant depletion of glutathione level. Besides, inflammatory cascades, including NF$x \mathrm{~B}$ and IL-6, were elevated significantly. Furthermore, 5-FU stimulated cell death through the significant increase of caspase-3. Interestingly, Pre-treatment with GH markedly ameliorated the deleterious effects of 5-FU through counteracting the oxidative stress and inflammation-mediated apoptosis. In addition, $\mathrm{GH}$ rescued the oral mucosal histology following chemotherapy.

Conclusions: Our study is the first to demonstrate evidences for the effective chemoprotection provided by $\mathrm{GH}$ against 5 -FU induced OM along with the underlying mechanisms, which may offer a promising adjuvant therapy for cancer patients.

KEYWORDS: Growth hormone, chemotherapy, 5- Fluorouracil, Oral mucositis, Inflammation.

* Oral Biology Department, Faculty of Dentistry, Al- Mansoura University.

** Pharmacology, Toxicology \& Biochemistry Department, Faculty of Pharmacy, Badr University in Cairo, Egypt.

*** Department of Pharmacology \& Toxicology, Faculty of Pharmacy, Ain Shams University, Cairo, Egypt. 


\section{INTRODUCTION}

Cancer is a remarkable health problem and a major cause of death worldwide and now considered as the single most important barrier to increasing life expectancy ${ }^{(1)}$. Although CT and RT are the most commonly used treatments for cancer patients, they are associated with several side effects, resulting in patient morbidity and mortality ${ }^{(2)}$. Oral mucositis represents one of the most enervating, symptomatic and worrying adverse effect of both $\mathrm{CT}$ and RT accompanied with marked morbidity and pain, in addition to odynodysphagia, dyseugia, and subsidiary dehydration and malnutrition ${ }^{(3)}$. The severity of mucositis varies from lesions with few symptoms to potential ulcers and pain that can affect any part of gastrointestinal tract from mouth to anus ${ }^{(4)}$. Severe $\mathrm{OM}$ can also alter the delivery of appropriate cancer therapy protocol, including dose adjustment or treatment scheduling, to permit for the resolution of oral lesions. An additional concern is that the patient may no longer be capable of enduring to cancer therapy, which can directly affect patient survivorship $^{(2)}$.

It is well established that $\mathrm{OM}$ is induced mainly by chemotherapeutic drugs affecting DNA synthesis $^{(5)}$. Indeed, 5-FU is a fluoropyrimidine widely used for different types of cancer, such as ovary ${ }^{(6)}$, breast ${ }^{(7)}$, gastrointestinal tract, head and neck ${ }^{(8)}$. It inhibits the thymidylate synthase, thus affecting the formation of thymine ${ }^{(9)}$. The OM associated with the use of 5 -FU occurs in more than $15 \%$ of $\operatorname{cases}^{(3)}$. The initial phase of mucositis was found to be attributable to an inflammatory reaction in response to chemotherapy-derived production of reactive oxygen species (ROS) ${ }^{(10)}$ in submandibular and sublingual glands ${ }^{(11)}$.

Despite the various treatment modalities for mucositis patients receiving $\mathrm{CT}$ or RT, none of them have proven highly effective ${ }^{(12)}$ Therefore, new treatment strategy is of great interest. Growth hormone is an anabolic hormone with multiple effects on cell growth, metabolism and differentiation. Growth hormone previously showed a synergistic protective effect combined with a hyperproteic diet against methotrexate-induced injury of intestinal mucosa ${ }^{(13)}$. Lately, concomitant administration of $\mathrm{GH}$ was found to have beneficial effects against radiation-induced intestinal mucositis, without affecting the tumor ${ }^{(14)}$. Consequently, our study targeted to illuminate the possible chemoprotective effects of GH in 5-FU-induced OM in rats, as well as investigating the possible underlying mechanisms.

\section{MATERIAL AND METHODS}

\section{Drugs and Chemicals:}

Recombinant human growth hormone (Genotropin, somatropin $1.3 \mathrm{mg} / 4 \mathrm{iU}$ inj.) from Pharmacia and Upjohn Co, Pfizer Inc. (NY). 5-Flourouracil (Fluorouracil Injection 250mg / 10ml Amp) from ACDIMA International (AiT). Glutathione reductase assay kit from Randox Laboratories (Antrim, UK). All other solvents and chemicals are of highest-grade and available commercially.

\section{Animals:}

Animal experiments follow the ARRIVE guidelines and executed in accordance with the National Institutes of Health guide for the care and use of Laboratory animals (NIH Publications No. 8023). In addition, the experimental protocol was approved by the Research Ethics Committee of Medical Faculty of Al Mansoura University, Egypt. Animals used in accordance with the Animal Welfare Act and Guide for Care Use of MERC (Mansoura Experimental Research Centre) prepared by Mansoura University. 32 Wister male rats (age $\approx 6-7$ weeks) weighing 150-200g, were acquired from the Nile Co. for Pharmaceutical and Chemical Industries, Egypt. Rats kept in an air-conditioned atmosphere, at a temperature of $25^{\circ} \mathrm{C}$ with $12 \mathrm{~h} \mathrm{light}$ and dark alternative cycles, and on a standard diet and water ad libitum. Before any experimentation, rats allowed to adapt for two weeks. 


\section{Experiment design:}

Rats were randomly assigned to 4 groups $(n=8)$ and treated for one week. Group 1: received only water for injection for 6 days (control). Group 2: received $100 \mathrm{mg} / \mathrm{kg}$ of 5-FU, i.p (on day 4). Groups 3: GH group received only $\mathrm{GH}(1 \mathrm{mg} / \mathrm{kg}$, s.c) for 6 days. Groups 4: received both $\mathrm{GH}+5 \mathrm{FU}$; $\mathrm{GH}$ $(1 \mathrm{mg} / \mathrm{kg}$ ) for 6 days; $\mathrm{GH}$ was given 3 days before 5-FU administration and 2 days after the first 5-FU injection, while 5-FU was given on day 4 . In the end of the study, blood samples were collected after anesthesia from the retro-orbital plexus, animals were euthanized using decapitation technique which produces prompt, painless unconsciousness. Using centrifugation, samples separated at $1000 \mathrm{~g}$ for $10 \mathrm{~min}$, then stored at $-80^{\circ} \mathrm{C}$. Lastly, rats were sacrificed, and oral mucosal tissues were dissected and saved in suitable buffers.

\section{Histopathological examination:}

Specimens from the buccal mucosa were kept in $10 \%$ neutral buffered formalin, then specimens were dehydrated in series of alcohol, cleared in xylene and embedded in molten paraffin. Using rotary microtome, 5 microns thickness sections were cut and mounted. Sections stained with hematoxylin and eosin for histopathological examination (H\&E).

\section{Oxidative stress markers assessment:}

Reduced glutathione (GSH) content was assayed in oral mucosal tissue homogenate using colorimetric kit (Sigma-Aldrich, St Louis, Missouri) based on previous method (15). Lipid peroxides (MDA) were assessed also in oral mucosa using colorimetric kit (OxiSelect ${ }^{\mathrm{TM}}$ TBARS assay Kit, Cell Biolabs, Inc.), by measuring the malondialdehyde (MDA) content as estimate for the thiobarbituric acid reactive substances (TBARS).

\section{Inflammation and Apoptosis assessment:}

Nuclear factor kappa $\mathrm{b} \quad(\mathrm{NF}-\varkappa \mathrm{B})$ and Caspase-3 oral mucosal contents were assessed immunohistochemically $(16,17)$. Tissue sections deparaffinized in xylene, then rehydrated by graded ethanol. Immunostaining for each applied marker was done according to abcam, USA, using an automated staining system (BioGenex i6000 systems). In addition, interleukin-6 (IL-6) was determined in oral mucosal tissue homogenate using ELISA kits (Elabscience Inc., USA).

\section{Digital morphometric study:}

Slides photographs were taken using Olympus ${ }^{\circledR}$ digital camera, which was installed on Olympus ${ }^{\circledR}$ microscope with a photo adaptor of $1 / 2 \mathrm{X}$. The intensity of immune stain was counted in 10 (non-overlapping) low power fields (LPF) at a magnification $(\times 250)$ in immunohistochemically stained sections. The analysis of images was done on Intel ${ }^{\circledR}$ Core $\mathrm{I} 3{ }^{\circledR}$ based computer by the use of Video Test Morphology® software (Russia) which has a specific built-in routine for area, area percentage and object counting. Staining intensity levels where determined as minimal, mild, moderate and strong immunoreactions.

\section{Statistics:}

GraphPad Prism software version 5 (ISI ${ }^{\circledR}$ ) software, USA) was used for the analysis and presentation of graphs. Data are represented as means \pm SD. Multiple group statistics were performed using ANOVA then by the Tukey-Kramer post-hoc test. The significance level was accepted at $\mathrm{P}<0.05$.

\section{RESULTS}

\section{Effect on body weight:}

Comparison between different groups regarding body weight changes revealed that there was a significant decrease in the animals' body weight of the 5-FU treated group by $17.5 \%$, compared to control group. Meanwhile, GH co-administration with 5-FU improved these changes notably by $9.4 \%$ when compared to 5-FU treated group (Fig.1). 




Fig. (1) Effect of GH treatment on body weight changes in 5-FU induced-oral mucositis. Data are expressed as $\%$ of initial body weight values (a or b: significantly different from the corresponding (control) or (5FU) group respectively at $\mathrm{P}<0.05)$.

\section{Histopathology of oral mucosa:}

The microscopical examination of different groups has been shown in (Fig.2), in which the control group revealed normal histological structure of the buccal mucosa with a stratified squamous keratinized epithelium \& underlying lamina propria. In addition, the submucosa muscular layers were clearly seen (A, A1). Meanwhile, 5- FU group exhibited a decrease in epithelial thickness with the detachment in-between the epithelial layers, areas of erosion and disorientation of the basal cell layer. In addition, connective tissue showed edema, dilatation of sub epithelial blood vessels, as well as few chronic cells infiltration (B, B1). In contrast, concurrent treatment with $\mathrm{GH}$ alleviated the pathological changes caused by 5-FU and showed normal epithelial thickness with intact epithelial layers with no evidence of eroded areas. The connective tissue showed normal blood vessels with tissue edema and absence of inflammatory cells (D, D1). However, normal rats treated with GH showed increase in the mucosal height and thickness (double arrow head), spongiosis of prickle cell layers with prominent nuclei, and epithelial cell proliferation are clearly seen with plump shaped rete pigs $(\mathrm{C}, \mathrm{C} 1)$.

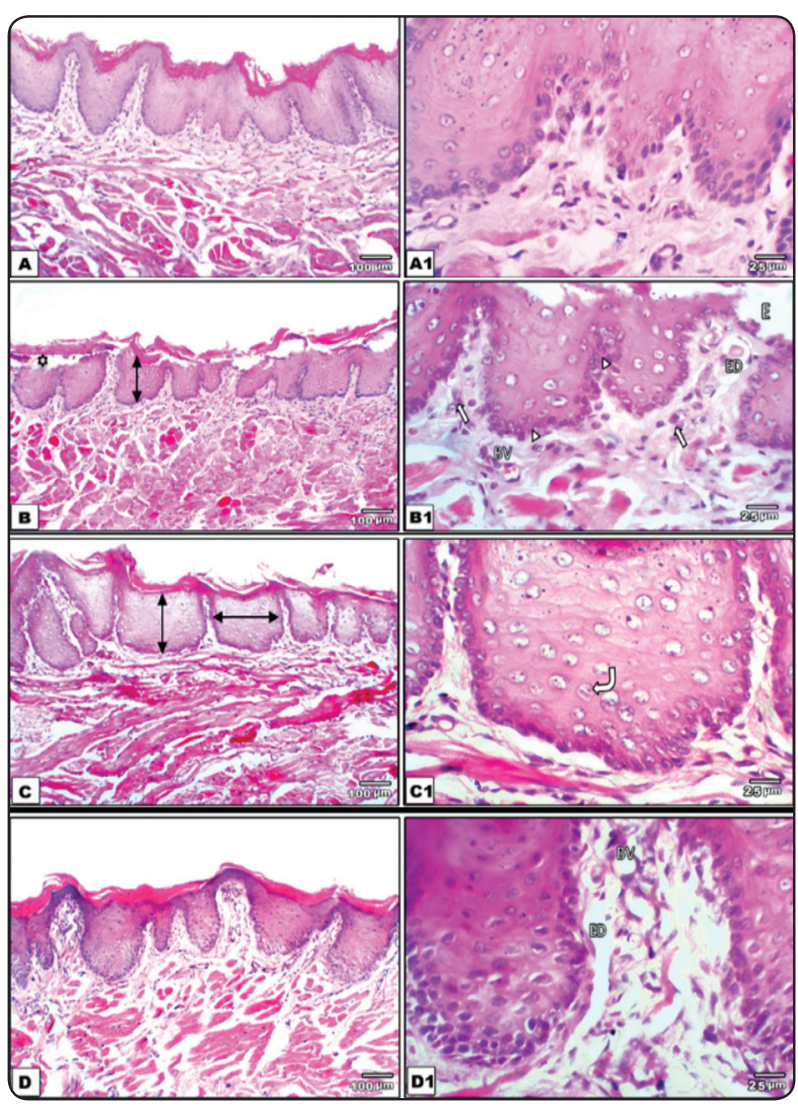

Fig. (2) Histological images of the buccal mucosa stained with H\&E. (A, A1) Control group: showing normal histological structure of the buccal mucosa with a stratified squamous keratinized epithelium \& underlying lamina propria as well as the submucosa muscular layers were clearly seen. (B, B1) 5-FU treated Group: exhibiting a decrease in epithelial thickness (double arrow head) with the detachment in between the epithelial layers (asterisk), areas of erosion (E), disorientation of the basal cell layer (arrow heads) and connective tissue showing edema (ED), dilatation of sub epithelial blood vessels (BV), as well as the few chronic cells infiltration (arrows). (C, C1) GH treated Group: showing increase in the mucosal height \& thickness (double arrow head), spongiosis of prickle cell layers with prominent nuclei (curved arrow). Epithelial cell proliferations are clearly seen with plump shaped rete pigs. (D, D1) 5-FU\&GH combined treated group: showing normal epithelial thickness with intact epithelial layers, no evidence of eroded areas. The connective tissue (CT) showing normal blood vessels (BV) with tissue edema (ED), and absence of inflammatory cells. 


\section{Anti-oxidant effects:}

Table (1) reveals a potential stimulation of oxidative stress in 5-FU treated group, showed by a significant decrease in GSH by $42.3 \%$ and a remarkable increase in MDA by $369.4 \%$, as compared to the control group. Meanwhile, concurrent treatment with $\mathrm{GH}$ significantly improved all of oxidative stress markers, interestingly by $83 \%$ in GSH and $46.4 \%$ in MDA, as compared to the 5-FU group (Table 1). Moreover, rats subjected to $\mathrm{GH}$ injections alone for 6 days have nonsignificant changes in GSH as well as MDA levels when compared to the control group.

TABLE (1): Effects of $\mathrm{GH}$ on oxidative stress markers in 5FU-induced oral mucositis.

\begin{tabular}{|c|c|c|}
\hline Groups & $\begin{array}{c}\text { GSH } \\
(\mathbf{m m o l} / \mathbf{g . t i s s u e})\end{array}$ & $\begin{array}{c}\text { MDA } \\
(\mathbf{n m o l} / \mathbf{g . t i s s u e})\end{array}$ \\
\hline Control & $26.5 \pm 2.6$ & $31.0 \pm 1.0$ \\
\hline $5 \mathrm{FU}$ & $15.3 \pm 1.3^{\mathrm{a}}$ & $145.5 \pm 6.4^{\mathrm{a}}$ \\
\hline $\mathrm{GH}$ & $25 \pm 2.0^{\mathrm{b}}$ & $38 \pm 3.5^{\mathrm{b}}$ \\
\hline $5 \mathrm{FU}+\mathrm{GH}$ & $28 \pm 1.4^{\mathrm{b}}$ & $78 \pm 2.8^{\mathrm{a}, \mathrm{b}}$ \\
\hline
\end{tabular}

-Data is represented as mean $\pm S D(n=8)$. a or b: significantly different from the corresponding (control) or $(5 F U+G H)$ group, respectively at $P<0.05$ using one-way ANOVA followed by Tukey-Kramer multiple comparison test.

\section{Anti-inflammatory and Anti-apoptotic effects}

Treatment with 5-FU induced a remarkable increase in inflammatory markers, including NFкB and IL-6, by $1124 \%$ and $70 \%$., respectively as compared to control rats. Concurrent administration of GH with 5-FU ameliorated these elevations significantly in by $61 \%$ and $44 \%$., respectively as compared to 5-FU treated group. Furthermore, GH showed moderate expression of NF- $x \mathrm{~B}$ in the connective tissue layer while epithelial layer was negative (Fig. 3 \& 4). Besides inflammation, Injection of 5-FU resulted in a marked induction of apoptosis as showed by $333 \%$ elevation in the activity of caspase-3, as compared to the control group. However, concomitant injection with GH normalized that increment by reducing caspase- 3 activity by about 58\%, when compared to 5-FU group (Fig. 5). Immunohistochemical reactivity for NF-kB and caspase- 3 was further assessed separately in the cytoplasm and in the nucleus of the epithelial cells. Positive areas were expressed as percentages of total analyzed area (Fig. 6I \& II, respectively).

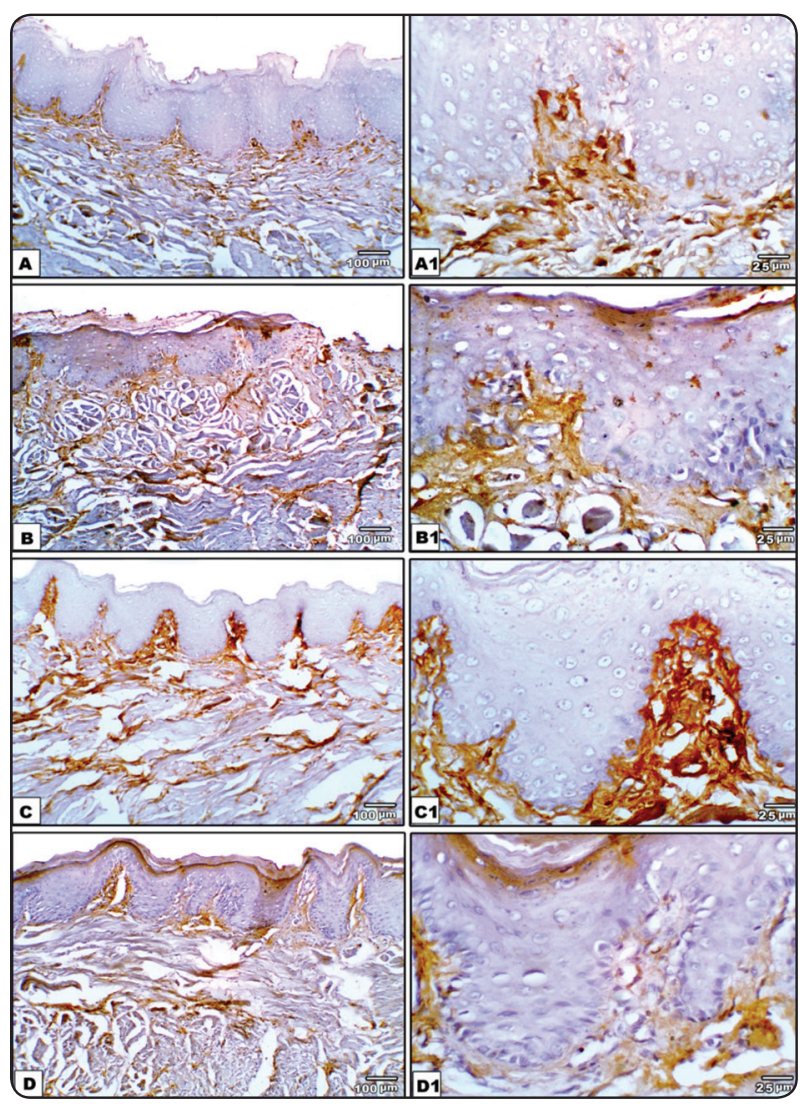

Fig.3 Immunohistochemical images of buccal mucosa stained with NF-kB antibody. (A,A1) Control group: showing negative immune reaction. (B,B1) 5-FU treated group: showing strong immune reaction. $(\mathrm{C}, \mathrm{C} 1) \mathrm{GH}$ treated group: showing moderate immune reaction (D,D1) 5-FU\&GH combined treated group: showing moderate reaction. 


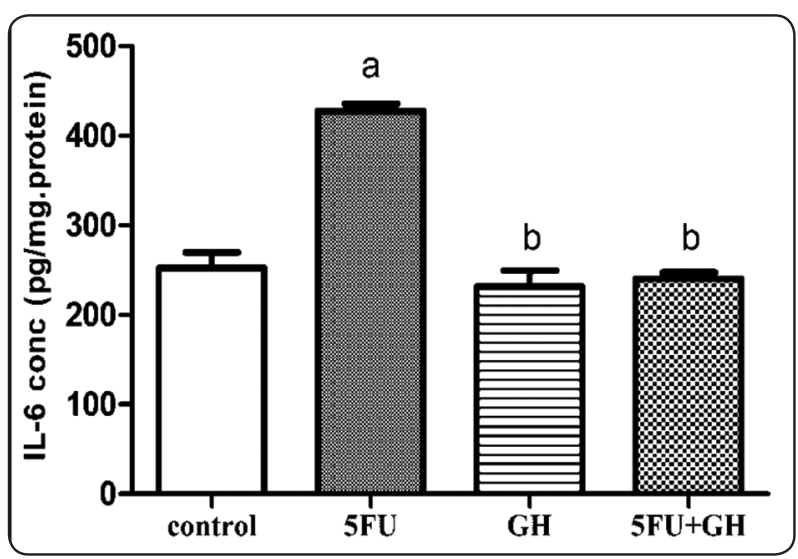

Fig. (4) Effect of GH treatment on IL-6 mucosal expression in 5-FU induced-oral mucositis. Data are represented as mean $\pm \mathrm{SD}$ (a or b: significantly different from the corresponding (control) or (5FU) group respectively at $\mathrm{P}<0.05)$.

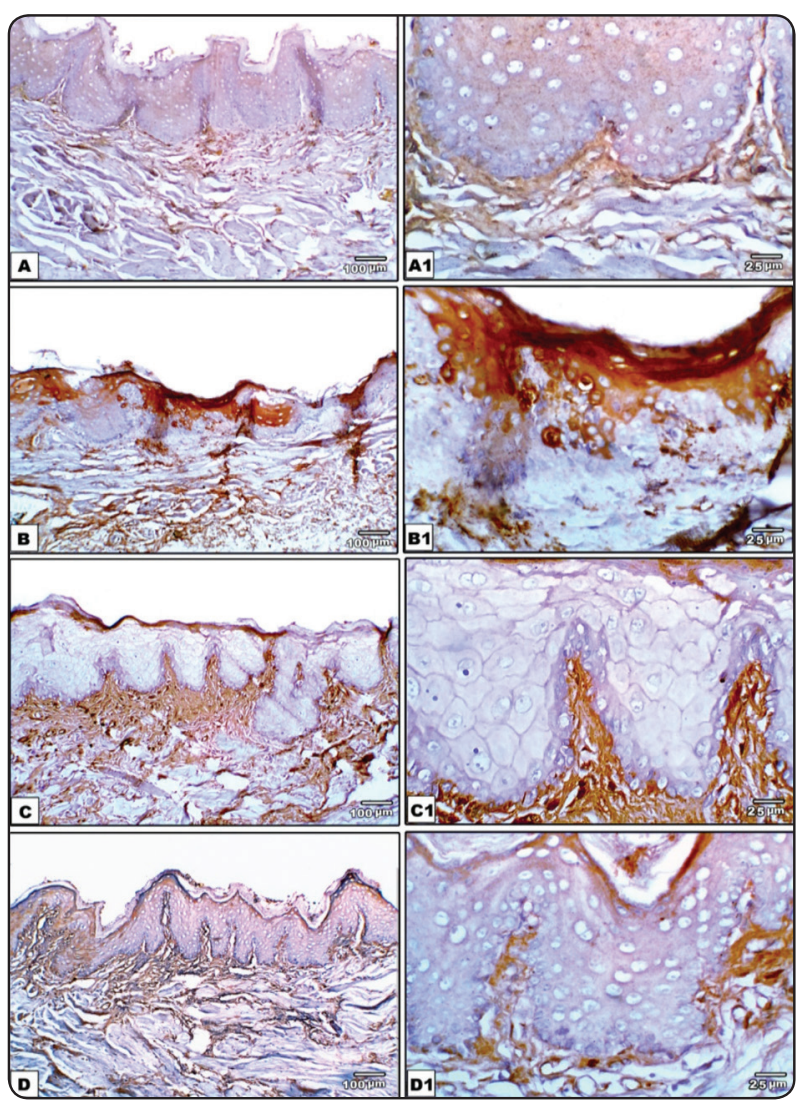

Fig. (5) Immunohistochemical images of buccal mucosa stained with caspase-3 antibody. (A,A1) Control group: showing negative immune reaction. (B,B1) 5-FU treated group: showing strong immune reaction. $(\mathrm{C}, \mathrm{C} 1)$ GH treated group: showing moderate immune reaction (D,D1) 5-FU\&GH combined treated group: showing moderate reaction.

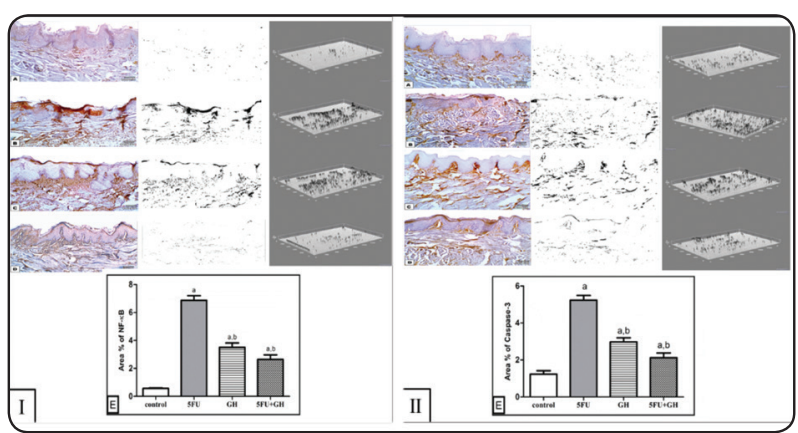

Fig. (6) Digital analysis of immunohistochemical images of buccal mucosa stained with NF-KB (I) or caspase-3 (II). (A) Control group: showing negative immune reaction, (B) 5-FU treated group: showing strong immune reaction, (C) $\mathrm{GH}$ treated group: showing moderate immune reaction, (D) 5-FU\&GH combined treated group: showing moderate reaction. (E) Positive areas measured and expressed as a percentage of total analyzed area (a or b: significantly different from the corresponding (control) or (5FU) group respectively at $\mathrm{P}<0.05$.).

\section{DISCUSSION}

The immanent common finding of the severe OM among cancer patients who are treated with CT boosts the immense need of an ideal chemoprotective agent that enhances the quality of life. Indeed, 5-flourrouracil, a standard cytotoxic agent, is one of most common chemotherapies associated with OM in cancer patients. This potential side effect threatens the efficacy of treatment by necessitating delay, dose reduction or drug discontinuation, in addition of the remarkable pain, morbidity and malnutrition (18). Patients receiving CT or RT were treated for mucositis with different strategies, including oral mouthwashes, analgesics, local anesthetics, and biological mucosal protection ${ }^{(12,19)}$. However, there is no "gold standard" therapy to prevent or treat oral mucositis at the present, thus OM along with its associated symptoms still represent an unmet insult (20). Consequently, the development of an effective strategy is of high priority in the oncological supportive care.

Growth hormone was previously reported to have a proliferative and protective actions against 
radiotherapy and methotrexate-induced intestinal injury ${ }^{(13,21)}$. Additionally, it is well-established that 5-FU injection induces mucositis in both oral mucosa and small intestine ${ }^{(22,23)}$, thus it is commonly used to investigate many aspects of oral mucositis, such as the pathophysiology, treatment effects and the mucotoxicity of used drugs ${ }^{(24)}$. The cytotoxicity of 5-FU impairs DNA replication, causing genome damage and programmed cell death ${ }^{(9)}$. Accordingly, the present study was designed to investigate the possible protective effects of $\mathrm{GH}$ against 5-FUinduced $\mathrm{OM}$.

Clinically, difficulty in eating and drinking has been reported in nearly $90 \%$ of patients receiving CT and RT, and the resultant body weight loss was approximately $85 \%{ }^{(25,26)}$. Our results revealed that 5-FU caused a remarkable decrease in the animals' body weight, while GH pre-treatment improved animals' appetite and restored their body weight. These results were in agreement with other experimental animal studies in which body weight showed significant reduction when subjected to 5 -FU ${ }^{(27)}$.

Buccal mucositis can affect any part of the gastrointestinal tract ${ }^{(4)}$. The pathogenesis of $\mathrm{OM}$ involves five phases; initiation, upregulation, signal amplification, ulceration and healing (28). In our study, histopathological examination confirmed that devastating events of OM was stimulated by the injection of 5-FU with a deterioration in epithelial, basal cell and connective layers, which were similarly shown in a previous study ${ }^{(29)}$. Our study revealed a significant improvement of all pathological changes induced by 5-FU upon concurrent treatment with $\mathrm{GH}$, confirming its protective effect.

Chemotherapy-induced $\mathrm{OM}$ is characterized by direct breaks in DNA strands in the basal epithelium, resulting in the release of ROS, leading to direct damage of cells ${ }^{(30)}$. The current study confirmed that administration of 5-FU induced a significant oxidative stress in oral mucosal tissue through enhancing lipid peroxidation, accompanied with a notable depletion of GSH. These results are consistent with previous studies which affirmed the induction of potential oxidative stress followed by 5 -FU injection, leading to cell death in oral mucosa ${ }^{(23,31)}$. Our findings of the reversal of these alterations by concomitant administration of $\mathrm{GH}$ are clear evidence of its inherent antioxidant activity.

Following the release of ROS, there is a subsequent production of pro-inflammatory cytokines, resulting in tissue damage and apoptosis. NF- $x \mathrm{~B}$, a key element in this process, upregulates other inflammatory mediators, therefore plays a key role in the mucositis apoptotic pathway ${ }^{\left({ }^{32}\right)}$. Additionally, $\mathrm{NF}-\boldsymbol{x B}$ activation precedes peaks in pro-inflammatory cytokines such as IL-6, in mucosal cells. Therefore $\mathrm{NF}-x \mathrm{~B}$ is considered the (gatekeeper) for the inflammatory pathways involved in mucositis ${ }^{(33)}$. The increases in IL-6 are also accompanied with the emergence of mucositis and a main factor in signaling pathways leading to tissue injury. This cascade potentiate the initial insult of mucositis through a positive feedback loop ${ }^{(34)}$.

In the present study, the inflammatory response was manifested by increased oral mucosal expression of NF- $x \mathrm{~B}$, along with the marked elevation in IL-6 tissue contents, indicating exaggerated inflammatory reaction during 5-FU induced mucositis. Meanwhile, concurrent administration with $\mathrm{GH}$ improved this inflammatory response, evidenced by the reduced expression of NF- $x \mathrm{~B}$, and subsequently the downstream inflammatory cascade by attenuating the expression of IL-6. Furthermore, an important previous finding confirmed that the inhibition of NF- $x \mathrm{~B}$ activity diminishes the chemo-resistance to 5-FU in human stomach cancer cell line ${ }^{(35,36)}$. Accordingly, GH might offer an enduring chemotherapeutic response if it is added to the 5-FU-based therapy.

Apoptosis in the basal layer of the oral mucosa is well known to occur in OM lesions ${ }^{(37)}$. In addition, ROS and inflammatory reactions may also induce the production of sphingomyelinase and ceramide 
synthase, resulting ceramide pathway activation leading to apoptosis ${ }^{(30)}$. In this regard, our study confirmed the significant cell death associated with 5-FU induced-OM, through the elevated caspase-3 in oral mucosal tissue. This insult was significantly reversed upon GH treatment, which confirms the role of GH in cell survival.

\section{CONCLUSIONS}

This study is the first to demonstrate the effective protection provided by $\mathrm{GH}$ against $5-\mathrm{FU}$ induced $\mathrm{OM}$ in vivo. This chemoprotective effect could be partially attributed to its anti-oxidant and anti-inflammatory activities, as well as hampering the apoptosis. Therefore, the present study may open a new scenario for the clinical usefulness of adding GH to chemotherapy-based treatment of cancer patients, to enhance both quality of life and treatment efficacy.

Despite the undeniable anti-apoptotic and anabolic effect of $\mathrm{GH}$, the exact mechanism by which tumor cells are unprotected remains unknown; thus, the threat of increased future cancer risk or tumor recurrence, impedes its clinical use in oncological patients. However, several data from long-term patient surveillance and meta-analysis studies suggest the safety of low-dose GH treatment, concluding that $\mathrm{GH}$ therapy does not increase the risk of cancer (38-40). Further investigations are highly recommended to establish the clinical applicability of $\mathrm{GH}$ in cancer patients treated with chemotherapy and to explore the possible interference with the anti-tumor activity of cytotoxic drugs.

\section{List of abbreviations:}

OM: Oral mucositis

RT: Radiotherapy

CT: Chemotherapy

5-FU: 5-flourouracil

GH: Growth hormone
NF- $\boldsymbol{\varkappa B}$ : Nuclear factor kappa B

IL-6: Interleukin 6

ROS: Reactive oxygen species

GSH: Reduced glutathione

MDA: Malondialdehyde

TBARS: Thiobarbituric acid reactive substances

\section{Declarations:}

\section{Ethics approval and consent to participate}

The experimental protocol was approved by the Research Ethics Committee of Medical Faculty of Al Mansoura University, Egypt. Animals used in accordance with the Animal Welfare Act and Guide for Care Use of MERC (Mansoura Experimental Research Centre) prepared by Mansoura University.

\section{Competing interests}

The authors declare that they have no competing interests.

\section{Authors' contributions}

All Authors equally contributed to the study.

\section{ACKNOWLEDGEMENTS}

This research did not receive any specific grant from funding agencies in the public, commercial, or not-for-profit sectors

\section{REFERENCES}

1. Siegel RL, Miller KD, Jemal A. Cancer statistics, 2018. CA: a cancer journal for clinicians. 2018;68(1):7-30.

2. Naidu MU, Ramana GV, Rani PU, Mohan IK, Suman A, Roy P. Chemotherapy-induced and/or radiation therapyinduced oral mucositis--complicating the treatment of cancer. Neoplasia (New York, NY). 2004;6(5):423-31.

3. Sonis ST, Elting LS, Keefe D, Peterson DE, Schubert M, Hauer-Jensen M, et al. Perspectives on cancer therapyinduced mucosal injury: pathogenesis, measurement, epidemiology, and consequences for patients. Cancer. 2004;100(9 Suppl):1995-2025. 
4. Scully C, Epstein J, Sonis S. Oral mucositis: a challenging complication of radiotherapy, chemotherapy, and radiochemotherapy. Part 2: diagnosis and management of mucositis. Head \& neck. 2004;26(1):77-84.

5. Al-Ansari S, Zecha JA, Barasch A, de Lange J, Rozema FR, Raber-Durlacher JE. Oral Mucositis Induced By Anticancer Therapies. Current oral health reports. 2015;2(4):202-11.

6. Watari H, Hosaka M, Mitamura T, Moriwaki M, Ohba Y, Todo Y, et al. Weekly paclitaxel/5-fluorouracil followed by platinum retreatment for patients with recurrent ovarian cancer: a single institution experience. European journal of gynaecological oncology. 2008;29(6):573-7.

7. Goncalves A, Pierga JY, Ferrero JM, Mouret-Reynier MA, Bachelot T, Delva R, et al. UNICANCER-PEGASE 07 study: a randomized phase III trial evaluating postoperative docetaxel-5FU regimen after neoadjuvant dose-intense chemotherapy for treatment of inflammatory breast cancer. Annals of oncology : official journal of the European Society for Medical Oncology. 2015;26(8):1692-7.

8. Mahipal A, Shibata D, Siegel E, Springett G, Almhanna K, Fulp W, et al. Phase I trial of combination of FOLFIRI and pasireotide, a somatostatin analogue, in advanced gastrointestinal malignancies. Investigational new drugs. 2015;33(5):1093-9.

9. Longley DB, Harkin DP, Johnston PG. 5-fluorouracil: mechanisms of action and clinical strategies. Nature reviews Cancer. 2003;3(5):330-8.

10. Sonis ST. The pathobiology of mucositis. Nature reviews Cancer. 2004;4(4):277-84.

11. Campos L, Nicolau J, Arana-Chavez VE, Simoes A. Effect of laser phototherapy on enzymatic activity of salivary glands of hamsters treated with 5-Fluorouracil. Photochemistry and photobiology. 2014;90(3):667-72.

12. Worthington HV, Clarkson JE, Bryan G, Furness S, Glenny AM, Littlewood A, et al. Interventions for preventing oral mucositis for patients with cancer receiving treatment. The Cochrane database of systematic reviews. 2011(4):CD000978.

13. Ortega M, Gomez-de-Segura IA, Vazquez I, Lopez JM, de Guevara CL, De-Miguel E. Effects of growth hormone plus a hyperproteic diet on methotrexate-induced injury in rat intestines. Acta oncologica (Stockholm, Sweden). 2001;40(5):615-21.
14. Caz V, Elvira M, Tabernero M, Grande AG, Lopez-Plaza B, de Miguel E, et al. Growth Hormone Protects the Intestine Preserving Radiotherapy Efficacy on Tumors: A Short-Term Study. PloS one. 2015;10(12):e0144537.

15. Fernandez-Checa JC, Kaplowitz N. Hepatic mitochondrial glutathione: transport and role in disease and toxicity. Toxicology and applied pharmacology. 2005;204(3):26373.

16. Kamperos G, Nikitakis N, Sfakianou A, Avgoustidis D, Sklavounou-Andrikopoulou A. Expression of NF-kappaB and IL-6 in oral precancerous and cancerous lesions: An immunohistochemical study. Medicina oral, patologia oral y cirugia bucal. 2016;21(1):e6-13.

17. Huang JS, Yang CM, Wang JS, Liou HH, Hsieh IC, Li GC, et al. Caspase-3 expression in tumorigenesis and prognosis of buccal mucosa squamous cell carcinoma. Oncotarget. 2017;8(48):84237-47.

18. Alvarino-Martin C, Sarrion-Perez MG. Prevention and treatment of oral mucositis in patients receiving chemotherapy. Journal of clinical and experimental dentistry. 2014;6(1):e74-80.

19. Yuan A, Sonis S. Emerging therapies for the prevention and treatment of oral mucositis. Expert opinion on emerging drugs. 2014;19(3):343-51.

20. Lalla RV. Alleviating mucositis: are we on track for a novel therapeutic? Expert review of gastroenterology \& hepatology. 2015;9(2):127-8.

21. Posadas SJ, Largo C, Merino JJ, Elvira M, Gonzalez $\mathrm{G}$, Caz V, et al. Growth hormone upregulates intestinal trefoil factor expression in the ileum of rats after gammaradiation. Experimental biology and medicine (Maywood, NJ). 2011;236(2):205-11.

22. Logan RM, Stringer AM, Bowen JM, Gibson RJ, Sonis ST, Keefe DM. Is the pathobiology of chemotherapyinduced alimentary tract mucositis influenced by the type of mucotoxic drug administered? Cancer chemotherapy and pharmacology. 2009;63(2):239-51.

23. Bomfin LE, Braga CM, Oliveira TA, Martins CS, Foschetti DA, Santos A, et al. 5-Fluorouracil induces inflammation and oxidative stress in the major salivary glands affecting salivary flow and saliva composition. Biochemical pharmacology. 2017;145:34-45.

24. Vanhoecke B, Bateman E, Mayo B, Vanlancker E, Stringer A, Thorpe D, et al. Dark Agouti rat model of chemotherapy- 
induced mucositis: establishment and current state of the art. Experimental biology and medicine (Maywood, NJ). 2015;240(6):725-41.

25. Cacicedo J, Casquero F, Martinez-Indart L, del Hoyo O, Gomez de Iturriaga A, Navarro A, et al. A prospective analysis of factors that influence weight loss in patients undergoing radiotherapy. Chinese journal of cancer. 2014;33(4):204-10.

26. Di Liberto C, Caroprese M, Pizzo G, Compilato D, Fulfaro $\mathrm{F}$, Rinaldi $\mathrm{T}$, et al. [Oral complications in patients with head and neck cancer after radio-chemotherapy. Mucositis and xerostomia]. Recenti progressi in medicina. 2007;98(5):302-14.

27. Song MK, Park MY, Sung MK. 5-Fluorouracil-induced changes of intestinal integrity biomarkers in BALB/c mice. Journal of cancer prevention. 2013;18(4):322-9.

28. Georgiou M, Patapatiou G, Domoxoudis S, PistevouGompaki K, Papanikolaou A. Oral Mucositis: understanding the pathology and management. Hippokratia. 2012;16(3):215-6.

29. Aras MH, Sezer U, Erkilic S, Demir T, Dagli SN. Effect of dietary boron on 5-fluorouracil induced oral mucositis in rats. European journal of dentistry. 2013;7(3):310-4.

30. Biswal BM. Current trends in the management of oral mucositis related to cancer treatment. The Malaysian journal of medical sciences : MJMS. 2008;15(3):4-13.

31. Yoshino F, Yoshida A, Nakajima A, Wada-Takahashi S, Takahashi SS, Lee MC. Alteration of the redox state with reactive oxygen species for 5-fluorouracil-induced oral mucositis in hamsters. PloS one. 2013;8(12):e82834.

32. Chang CT, Ho TY, Lin H, Liang JA, Huang HC, Li CC, et al. 5-Fluorouracil induced intestinal mucositis via nuclear factor-kappaB activation by transcriptomic analysis and in vivo bioluminescence imaging. PloS one. 2012;7(3):e31808.
33. Logan RM, Gibson RJ, Sonis ST, Keefe DM. Nuclear factor-kappaB (NF-kappaB) and cyclooxygenase-2 (COX-2) expression in the oral mucosa following cancer chemotherapy. Oral oncology. 2007;43(4):395-401.

34. Sonis ST. Pathobiology of oral mucositis: novel insights and opportunities. The journal of supportive oncology. 2007;5(9 Suppl 4):3-11.

35. Uetsuka H, Haisa M, Kimura M, Gunduz M, Kaneda Y, Ohkawa T, et al. Inhibition of inducible NF-kappaB activity reduces chemoresistance to 5-fluorouracil in human stomach cancer cell line. Experimental cell research. 2003;289(1):27-35.

36. Li F, Sethi G. Targeting transcription factor NF-kappaB to overcome chemoresistance and radioresistance in cancer therapy. Biochimica et biophysica acta. 2010;1805(2): $167-80$.

37. GIBSON RJ, CUMMINS AG, BOWEN JM, LOGAN RM, HEALEY T, KEEFE DM. Apoptosis occurs early in the basal layer of the oral mucosa following cancer chemotherapy. Asia-Pacific Journal of Clinical Oncology. 2006;2(1):39-49.

38. Child CJ, Zimmermann AG, Woodmansee WW, Green DM, Li JJ, Jung H, et al. Assessment of primary cancers in GH-treated adult hypopituitary patients: an analysis from the Hypopituitary Control and Complications Study. European journal of endocrinology. 2011;165(2):217-23.

39. Wilton P, Mattsson AF, Darendeliler F. Growth hormone treatment in children is not associated with an increase in the incidence of cancer: experience from KIGS (Pfizer International Growth Database). The Journal of pediatrics. 2010;157(2):265-70.

40. Shen L, Sun CM, Li XT, Liu CJ, Zhou YX. Growth hormone therapy and risk of recurrence/progression in intracranial tumors: a meta-analysis. Neurological Sciences. 2015;36(10):1859-67. 